UDK $674.03: 542.943$

\title{
KINETICS OF FIR WOOD OXIDATION BY OXYGEN IN AQUEOUS
}

\section{ALKALINE MEDIA}

\author{
(C) V.E. Tarabanko ${ }^{1,2^{*}}$, E.P. Pervishina ${ }^{2}$, N.V. Tarabanko ${ }^{1}$, M.Yu. Chernyak ${ }^{1}$, K.L. Kaygorodov ${ }^{1}$, \\ Yu.V. Chelbina ${ }^{1}$, D.V. Boyarchuk ${ }^{2}$ \\ ${ }^{1}$ Institute of Chemistry and Chemical Technology SB RAS, Federal Research Center \\ "Krasnoyarsk Science Center SB RAS", Akademgorodok, 50/24, Krasnoyarsk, \\ 660036 (Russia), e-mail: veta@icct.ru \\ ${ }^{2}$ Siberian Federal University, Svobodny pr., 79, Krasnoyarsk, 660041 (Russia)
}

\begin{abstract}
Kinetics of fir wood oxidation (Abies sibirica) in aqueous alkaline media were studied by measuring oxygen consumption rates. Oxidation of extracted wood conforms to the kinetics of non-branching chain mechanism. The same mechanism governs the initial stage of non-extracted wood oxidation, but a transition to degenerate branching mode soon occurs. This transition is probably caused by softwood's low hemicelluloses content and high content of propyl guaiacol lignin units that are prone to condensation involving phenolic hydroxyls. This leads to quick depletion of phenolic and uronic carboxylic groups; chain initiation by oxidizing the respective anions becomes impossible, and degenerate branching takes the lead. It is assumed that inert hydrophobic fir extractives physically shield hydroperoxides responsible for degenerate branching from alkali. This prevents alkali from causing heterolytic hydroperoxide cleavage that does result in chain branching. It is assumed that the catalyst accelerates the reaction of homolytic cleavage of the aforementioned hydroperoxides.

Keywords: Wood chemistry, kinetics, fir, Abies sibirica, oxidation, oxygen delignification, wood extractives.

Financing. The reported study was funded by RFBR, Government of Krasnoyarsk Territory, and Krasnoyarsk Territory Scientific Foundation according to the research project № 16-43-242102.
\end{abstract}

\section{Introduction}

Oxidation of ligneous materials by oxygen is an economical and environmentally benign approach to pulp bleaching. If catalysts are used, this process can be applied for wood delignification [1]. Catalytic oxidation of wood in alkaline media produces valuable aromatic aldehydes (vanillin and syringaldehyde); recently, this particular reaction was evaluated as a way of isolating cellulose (e.g., suitable for enzymatic hydrolysis) [2]. Improving

Tarabanko Valery Evgenievich - Head of the Laboratory for Comprehensive Biomass Processing, Doctor of Chemical Sciences, Professor, e-mail: veta@icct.ru

Pervyshina Ekaterina Petrovna - Candidate of Chemical Science, e-mail: veta@icct.ru

Tarabanko Nikolay Valerievich - Researcher in the Laboratory for Comprehensive Biomass Processing, Candidate of Chemical Science, e-mail: quartz8585@yandex.ru Chernyak Mikhail Yurievich - Senior Researcher in the Laboratory for Comprehensive Biomass Processing, Candidate of Chemical Science, e-mail: chernyak@icct.ru Kaygorodov Konstantin Leonidovich - Junior Researcher in the Laboratory for Comprehensive Biomass Processing, e-mail:kulik@icct.ru

Chelbina Yulia Vyacheslavovna - Junior Researcher in the Laboratory for Comprehensive Biomass Processing, e-mail: agafon5@mail.ru

Boyarchuk Dmitry Valentinovich - Student, e-mail: veta@icct.ru productivity of the described processes and quality of thereby obtained products requires detailed understanding of the mechanisms and kinetics of the underlying chemical reactions.

Numerous investigations have been devoted to oxidation of residual lignin in technical-grade pulps and of lignin model compounds [3]. However, oxidation of whole wood by oxygen attracts considerably less attention [4], particularly in alkaline media (the latter situation can be explained by limited stability of the carbohydrate portion of wood in alkali at high temperature [5]). There exist studies of aspen wood oxidation in alkaline media without catalysts and with copper hydroxide [6]. These studies show that this process can proceed without diffusional limitations; they also suggest the radical chain mechanism of the

\footnotetext{
* Corresponding author
} 
oxidation reaction (short chains in strongly alkaline media, long chains at $\mathrm{pH} 10-12$, degenerate branching chains at lower basicity).

Similar studies on the kinetics of softwood oxidation in aqueous alkaline media are not described in scientific literature. Nonetheless, there is practical interest in oxidation of softwood. This type of wood constitutes the majority of lumber industry feedstock. Oxidation of softwood lignins yields just one aromatic aldehyde (vanillin), which eliminates the need of separating it from syringaldehyde (that would also form when oxidizing hardwood [7]).

Qualitative composition of softwood extractive components is quite distinctly different from those of hardwood. Softwood components contain a considerable portion of neutral non-hydrolyzable terpenoids, whereas hardwoods contain mostly esters of long-chain fatty acids [8]. The presence of inert hydrophobic zones formed by softwood extractives in reaction media during softwood oxidation might affect the process kinetics. This subject gains more significance in the context of a recent paper that proposes oxidative delignification of pine wood with simultaneous vanillin synthesis [2]. That work studied the oxidation of extracted wood. However, effective oxidation of lignin into vanillin and preserving cellulose in the reaction media are two requirements that counteract each other: Vanillin formation requires harsh conditions that are destructive to wood carbohydrates. Therefore, attaining good yields of both vanillin and cellulose requires finely tuned reaction conditions. The presence of extractives in a reactor might influence the process kinetics, and affect its economic prospects.

The goal of the present study is to experimentally investigate the kinetics of oxidizing fir (Abies sibirica) wood by oxygen in aqueous alkaline media, along with the influence of the wood extractives on this process. In this work, we use oxygen consumption rates as the main kinetics descriptor, as employed in our previous work [6]. This approach is a simple way of continuously monitoring the reaction dynamics, and it enables direct comparison with the results of aspen wood oxidation.

\section{Experimental}

Sawdust of fir wood (Abies sibirica) was used as the feedstock for experiments. Wood composition: 50,3 wt.\% cellulose, $15,4 \%$ hemicelluloses, $27,7 \%$ lignin, $6,3 \%$ extractives. Removal of the extractives for the experiments with extracted substrate was accomplished according to a standard method [9].

The kinetic measurements were carried out in a stainless-steel reciprocating rocking autoclave $(100 \mathrm{ml} \mathrm{ca}-$ pacity) equipped with a pressure measurement system to monitor the oxygen consumption throughout the process [10]. The reactor was fed with oxygen from a calibrated buffer through a manually operated valve, and the oxygen consumption during the process was calculated from the pressure decrease in the buffer volume. Initial $\mathrm{pH}$ in the experiments was varied between 8,0 and 12,5. Phosphate and carbonate buffers (the total concentration of potassium salts was $300 \mathrm{~g} / \mathrm{L}$ ) as well as a $\mathrm{NaOH}$ solution were used to stabilize $\mathrm{pH}$ in the experiments. The difference in the reaction media acidity between the initial and final points of the kinetic curves did not exceed $0,2 \mathrm{pH}$ units.

$2 \mathrm{~g}$ of wood charge was used for each experiment, reactor rocking frequency was $6 \mathrm{~s}^{-1}$. As previously established, these conditions minimize the influence of diffusional limitations on the process [6].

The reactor was charged with a reaction mixture comprising a wood sample and a buffer solution. If a catalyst was necessary for an experiment, it was added in the form of copper sulfate aqueous solution. The volume of each reaction mixture was $60 \mathrm{ml}$. Then, the reactor was sealed and flushed with oxygen. Within 7-10 min of heating, the necessary temperature was attained. Afterwards, the reactor motor was turned on. Oxygen at the required pressure was introduced from the buffer volume through a manually operated valve. As the reaction was proceeding, the oxygen pressure drop due to its consumption was compensated by introducing more oxygen from the buffer volume.

\section{Results and discussion}

Figure 1 presents the dependences of reacted oxygen amount versus duration of non-catalytic oxidation of non-extracted fir wood at different oxygen partial pressures. Overall shapes of these dependences are very different from those obtained in oxidation of aspen wood under similar conditions [6]. Oxidation of aspen wood shows no sign of slowing down when oxygen consumption $50 \mathrm{~g}$ per $\mathrm{kg}$ of wood is attained. On the other hand, oxidation of fir exhibits a fast initial stage, but upon attaining oxygen consumption $15-25 \mathrm{~g} / \mathrm{kg}\left(0,03-0,05 \mathrm{~g} \mathrm{O}_{2}\right.$ per batch), the reaction slows down quite abruptly and afterwards proceeds at mostly constant rate. 
Figure 2-a shows the calculation of the reaction orders in oxygen (pre-kink and post-kink stages) and in hydroxyl ion (post-kink) for non-extracted wood oxidation. During the pre-kink stage, the order in oxygen is 0,49 which coincides with the value for aspen wood oxidation [6]. When the fir oxidation slowdown occurs, the reaction order in $\mathrm{O}_{2}$ decreases to 0,17 , the order in $\mathrm{OH}^{-}$is 0,13 .

In the context of the kinetic model underlying the earlier work [6], the decrease of the reaction order in oxygen from $\approx 0,5$ to near-zero values corresponds to the transition from non-branching long-chain mechanism to degenerate branching mechanism driven by homolytic cleavage of the organic hydroperoxides that accumulate in the reaction medium during oxidation. The increase in the role of degenerate branching also manifests in low reaction order in hydroxyl ion: Calculated from the post-kink linear sections of kinetic curves, the order in $\mathrm{OH}^{-}$is 0,13 . Therefore, in the process of non-extracted fir wood oxidation, degenerate branching reactions manifest early, which is significantly different from aspen wood oxidation.

Figure 1. Consumption of oxygen during oxidation of non-extracted fir wood by oxygen without catalyst. The legend details oxygen partial pressure.

Temperature $110^{\circ} \mathrm{C}, \mathrm{pH} 11,6$. The symbols represent experimental data, the lines are for the ease of perception and do not represent the process dynamics in any way
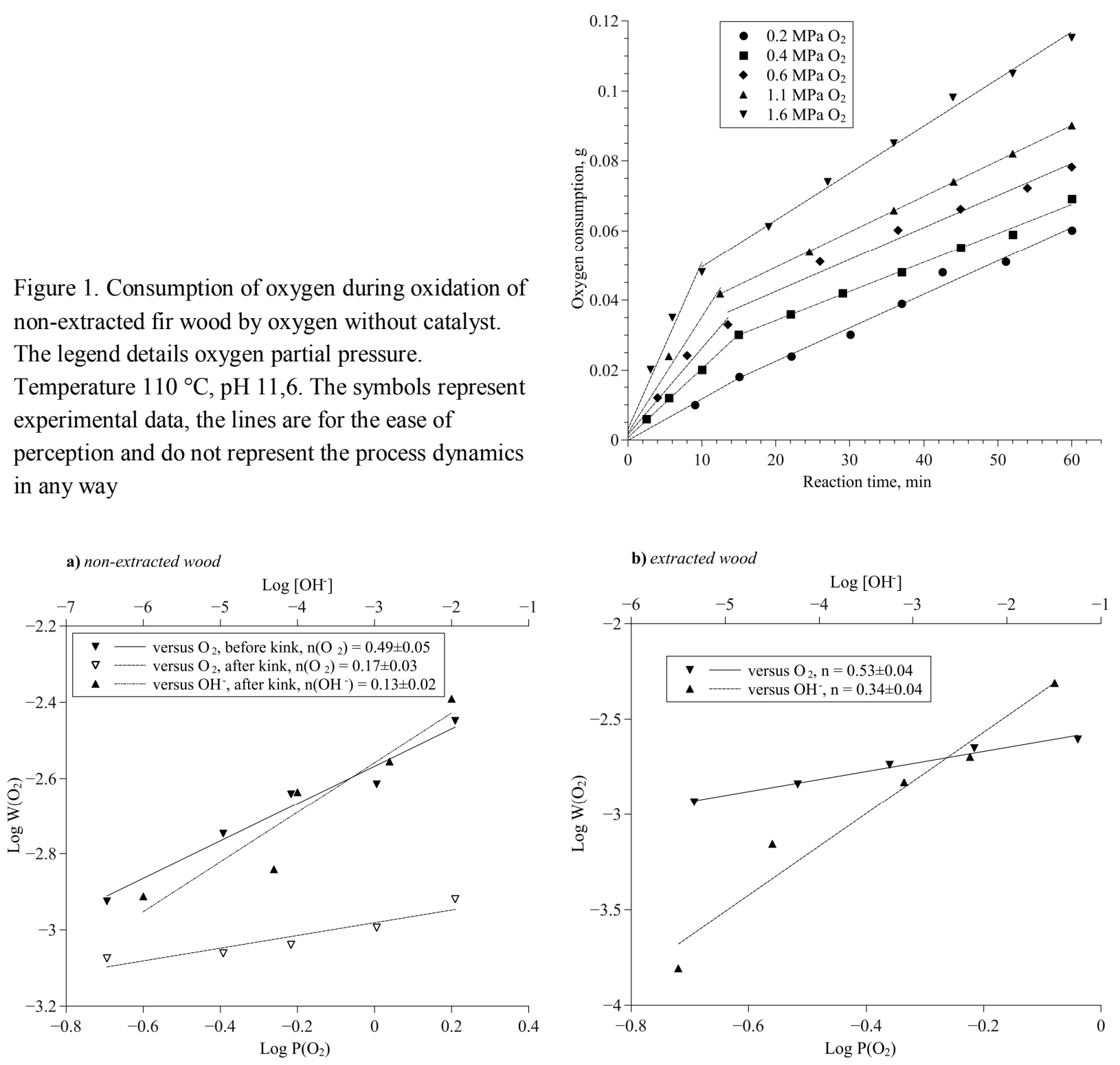

Figure 2. Logarithmic dependences of oxygen consumption rate versus reactant concentrations for the reaction of fir wood oxidation, temperature $110^{\circ} \mathrm{C}$. Symbols represent experimental data, lines represent linear fits thereof. a) oxidation of non-extracted fir wood, $\mathrm{n}\left(\mathrm{O}_{2}\right)=0,49 \pm 0,05$ before the kinetic curves kink in Fig. 1 (initial 15 min of the process), $\mathrm{n}\left(\mathrm{O}_{2}\right)=0,17 \pm 0,03$ after the kink (15-60 min time frame in Fig. 1), $\mathrm{n}\left(\mathrm{OH}^{-}\right)=0,13 \pm 0,02$ after the kink. b) oxidation of extracted fir wood, $\mathrm{n}\left(\mathrm{O}_{2}\right)=0,53 \pm 0,04, \mathrm{n}\left(\mathrm{OH}^{-}\right)=0,34 \pm 0,04$ 
In non-extracted fir wood oxidation, the absolute oxygen consumption rate decreases after the transition to degenerate branching mechanism. Within the kinetic model used in the previous work [6], oxygen consumption rate depends - caeteris paribus - on the rate of chain initiation. Thus, on one hand, at the onset of the reaction slowdown, in the rate of chain initiation there is significant increase of the role of degenerate branching driven by homolytic hydroperoxide cleavage. On the other hand, the overall rate of chain initiation decreases. I.e., as the reaction of fir oxidation is proceeding, there is rapid decrease in the concentration of compounds that drive the chain initiation which is independent of hydroperoxides. Apparently, in the herein described case, it is the decrease in the amount of phenolic hydroxyls and hemicellulosic uronic carboxyls. In other words, the amount of these functional groups in fir wood is considerably lower than in aspen wood.

This assumption finds justification in the known differences of composition of hardwood and softwood timbers. Softwood lignins comprise predominantly propyl guaiacol units (as opposed to a significant portion of propyl syringol units in hardwood lignins [7]). Propyl guaiacol units have an unsubstituted hydrogen atom in ortho position to the hydroxyl group, which makes them vulnerable to condensation reactions, including the $\beta-O-4$ and 5-O-4 condensations that lead to depletion of free phenolic hydroxyls. Moreover, these condensation reactions result in steric hindrance of phenolic hydroxyls, that can lead to lower rate of phenolate ions oxidation, i.e., lower chain initiation rate. Another factor that leads to the kinetic differences in oxidation of fir and aspen wood is the differences in the amount and composition of hemicelluloses [11]. Fir wood contains 2-3 times less of the most reactive pentosic hemicelluloses formed by xylose and arabinose.

Apparently, the principal pathway of degenerate chain branching is homolytic cleavage of hydroperoxides catalyzed by ions and/or complexes of transition metals (either found in the ash components of wood, or etched from reactor walls). E.g., this influence of transition metals is the reason why the addition of o-phenanthroline accelerates oxygen delignification [12]. The conclusion about the defining role of the catalytic pathway of hydroperoxide decomposition can be made by estimating the rates of catalytic and non-catalytic peroxide decomposition. The estimates made hereinafter are based on the reference data on the rate constants for reactions involving substances like hydroperoxides of cumene, isobutane, etc. [13]. It can be shown that, e.g., at $110^{\circ} \mathrm{C}$, hydroperoxide concentration $0,07 \mathrm{M}$, and substrate $\mathrm{R}_{\mathrm{s}} \mathrm{H}$ concentration $1 \mathrm{M}$, the rate of non-catalytic decomposition $(1,2)$ is within the interval $10^{-9}-10^{-7} \mathrm{M} / \mathrm{s}$. However, in the presence of $10^{-4} \mathrm{M} \mathrm{Fe} e^{2+}$, the rate of cumene hydroperoxide decomposition in aqueous medium $(3,4)$ is on the order of magnitude of $10^{-2} \mathrm{M} / \mathrm{s}$. This means that even trace amounts of transition metals suffice in order for the catalytic hydroperoxide cleavage pathway to dominate over the non-catalytic one.

$$
\begin{gathered}
\mathrm{ROOH}+\mathrm{ROOH} \rightarrow \mathrm{RO}_{2} \bullet+\mathrm{RO} \bullet+\mathrm{H}_{2} \mathrm{O} \\
\mathrm{ROOH}+\mathrm{R}_{\mathrm{s}} \mathrm{H} \rightarrow \mathrm{RO} \bullet+\mathrm{R}_{\mathrm{s}} \bullet+\mathrm{H}_{2} \mathrm{O} \\
\mathrm{ROOH}+\mathrm{M}^{\mathrm{n}+} \rightarrow \mathrm{RO} \bullet+\mathrm{OH}^{-}+\mathrm{M}^{(\mathrm{n}+1)+} \\
\mathrm{ROOH}+\mathrm{M}^{(\mathrm{n}+1)+} \rightarrow \mathrm{RO}_{2} \bullet+\mathrm{H}^{+}+\mathrm{M}^{\mathrm{n}+}
\end{gathered}
$$

It should be noted that in alkaline aqueous media, heterolytic, non-radical hydroperoxide cleavage is possible; this pathway does not lead to the oxidation chain reaction branching. The work of Skibida et al. [14] shows that in the presence of alkali, the non-radical hydroperoxide decomposition is predominant, with homolytic cleavage contributing only $0,01 \%$ to the overall decomposition rate. Retardation of homolytic cleavage by bases becomes more effective the stronger the base is and the more acidic is the $\alpha-\mathrm{C}-\mathrm{H}$ bond in the hydroperoxide [14]. The outlined non-radical destruction of hydroperoxides may be a factor leading to predominantly non-branching oxidation of aspen wood in alkaline media ( $\mathrm{pH} 9,6-12,5)$ [6].

If the reaction medium contains areas that are inaccessible to alkali, then chain-branching-promoting homolytic hydroperoxide cleavage will be more likely within these areas. Such hydrophobic zones can be represented by particles of fir extractives that are quite abundant in the timber (6-7 wt.\%). As earlier described in the Introduction, fir extractives predominantly comprise hydrophobic substances that are more or less chemically inert under the reaction conditions, whereas hardwood extractives are primarily represented by hydrolyzable esters. The latter are unstable in alkaline reaction media, and cannot isolate hydroperoxides from the alkaline aqueous phase 
(i.e., cannot prevent non-radical destruction of hydroperoxides). So, the outlined differences in composition of wood extractives between fir and aspen can explain the kinetic differences in oxidation of these two timbers.

In order to test the assumption about the role of extractives in fir wood oxidation, experiments with extracted material were conducted. Figure 2-b shows the logarithmic dependences of oxygen consumption rates versus hydroxyl ion concentration and oxygen partial pressure obtained in these experiments. Removal of the extractives results in the reaction order in $\mathrm{OH}^{-}$and $\mathrm{O}_{2}$ increasing to $0,34 \pm 0,04$ and 0,53 $\pm 0,04$ respectively. The increase of the reaction orders to the level typical for non-branching long chain reactions caused by the removal of fir extractives confirms the hypothesis about the role of softwood terpenoids serving as the hydrophobic phase that is inaccessible to aqueous alkali, and thus provides a 'safe zone' for homolytic cleavage of hydroperoxides that drives degenerate chain branching.

To summarize the talk about the mechanisms of hardwood and softwood oxidation, it can be said that when oxidizing either aspen or fir wood, at low conversions (oxygen consumption up to 15-25 g per kg of wood) longchain non-branching mechanism can be observed. With aspen wood, this mechanism also remains dominant at high conversions, and the contribution of degenerate branching manifests only at low $\mathrm{pH}$ or when delignification extent reaches dozens of percents. With fir wood, even at high $\mathrm{pH}$ degenerate branching becomes the dominant chain initiation pathway soon (oxygen consumption above $15-25 \mathrm{~g} / \mathrm{kg}$ ), but it can be suppressed by removing the extractives from the wood.

Figure 3 presents Arrhenius plots of oxygen consumption rate in fir wood oxidation with a catalyst (copper hydroxide) and without such. The apparent activation energy of fir oxidation is practically unaffected by the addition of the catalyst $(57-63 \mathrm{~kJ} / \mathrm{mol})$. This phenomenon can be explained in the context of the herein above described hypothesis about degenerate branching process mechanism in which chain initiation by branching dominates over initiation via phenolate ion oxidation. As detailed above, even when no extraneous catalyst is added to the system, chain branching is still very likely to be a catalytic reaction (catalyzed by miscellaneous impurities of transition metals). Therefore, the nature of the chain initiation stage is unaffected by deliberate catalyst addition. Adding a catalyst only increases the rate of chain initiation via hydroperoxide homolytic cleavage, the reaction that is in practice catalytic anyway because of unavoidable metal presence-from wood ash and/or etched from reactor walls. From this viewpoint, apparent activation energies of processes with and without deliberate catalyst addition can be very close to each other, and this situation is indeed observed in our experiments. A paper by Hermer [15] also demonstrates that oxidation of aspen wood in degenerate branching mode is characterized by the independence of apparent activation energy from catalyst addition; $E_{a}$ is $72-73 \mathrm{~kJ} / \mathrm{mol}$ which is similar to the values obtained in the present research.

The experimentally obtained apparent activation energies can be analyzed semi-quantitatively based on reference data about radical chain oxidation of hydrocarbons. According to [16], rate of oxygen consumption in a chain oxidation reaction depends on chain initiation rate and substrate concentration:

$$
\mathrm{W}=\mathrm{k}_{\mathrm{p}} \mathrm{C}_{\mathrm{s}}\left(\mathrm{W}_{\mathrm{i}} / \mathrm{k}_{\mathrm{t}}\right)^{1 / 2}
$$

where $\mathrm{k}_{\mathrm{p}}$ and $\mathrm{k}_{\mathrm{t}}$ are rate constants of chain propagation and termination respectively, $\mathrm{W}_{\mathrm{i}}$ is rate of chain initiation, $\mathrm{C}_{\mathrm{s}}$ is substrate concentration. In logarithmic form, Equation (5) may be transformed into the relation between apparent activation energy and activation energies of individual process stages:

$$
\mathrm{E}_{\mathrm{a}-\mathrm{app}}=\mathrm{E}_{\mathrm{a}-\mathrm{p}}+\mathrm{E}_{\mathrm{a}-\mathrm{i}} / 2-\mathrm{E}_{\mathrm{a}-\mathrm{t}} / 2
$$

where $E_{a-a p p}, E_{a-p}, E_{a-i}, E_{a-t}$ are activation energies of overall process, propagation stage, initiation stage, and termination, respectively. Activation energy of chain propagation $\mathrm{E}_{\mathrm{a}-\mathrm{p}}$ is typically in the $30-50 \mathrm{~kJ} / \mathrm{mol}$ range, chain termination activation energies are 10-30 kJ/mol [16]. Activation energy of chain initiation by hydroperoxide cleavage is considerably greater, $70-117 \mathrm{~kJ} / \mathrm{mol}$ without catalysts and $40-115 \mathrm{~kJ} / \mathrm{mol}$ with catalysts [13]. According to Equation (6), apparent activation energy of chain reactions can be in a rather wide range $45-95 \mathrm{~kJ} / \mathrm{mol}$; our experimental values $57-63 \mathrm{~kJ} / \mathrm{mol}$ belong to the middle of this range. The apparent activation energies obtained in the present work further confirm the hypothesis about degenerate branching chain mechanism of fir wood oxidation. 
Figure 3. Arrhenius plots of oxygen consumption rates in the reaction of fir wood oxidation in alkaline aqueous media. Catalyst concentration $8,2 \mathrm{~g} / \mathrm{l}$ of $\mathrm{CuO}$ equivalent, oxygen pressure $0,8 \mathrm{MPa}$. Catalytic process at hydroxyl concentration $2 \mathrm{M}$ has $\mathrm{E}_{\mathrm{a}}=57,8 \pm 2,5$. Non-catalytic process at hydroxyl concentration $2 \mathrm{M}$ has $\mathrm{E}_{\mathrm{a}}=61,3 \pm 6,3$. Non-catalytic process at hydroxyl concentration $3 \mathrm{mM}$ has $\mathrm{E}_{\mathrm{a}}=56,8 \pm 8,3$

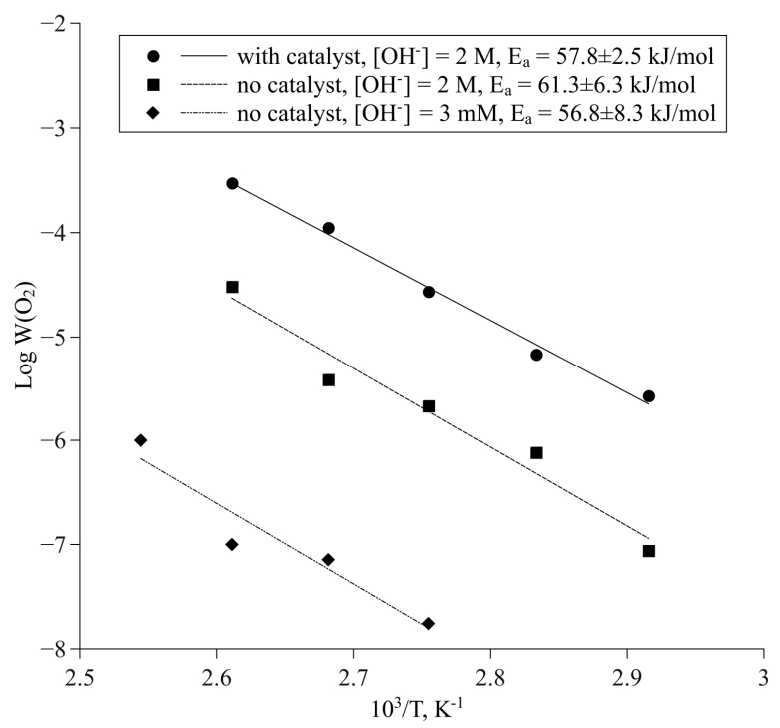

It can also be noted that activation energies of non-catalytic $(1,2)$ and catalytic $(3,4)$ hydroperoxide cleavage tend to be close to each other. E.g., activation energies of cumene hydroperoxide decomposition without catalyst and with copper phthalocyanine are practically equal (approximately $115 \mathrm{~kJ} / \mathrm{mol}$ ) [13]. This suggests that deliberately introducing a catalyst into a reaction system might not notably affect the apparent activation energy even if the reaction system in question does not initially contain enough miscellaneous metal impurities to appreciably catalyze hydroperoxide homolytic cleavage.

\section{Conclusion}

The studied kinetic parameters of fir wood oxidation in alkaline aqueous media can be compared to those of aspen wood oxidation $[6,17]$ (Table).

While the chemical reactions involved in oxidation of both wood species are practically identical, considerable differences in their composition lead to notable differences in the process kinetics. Fir wood has lower content of hemicellulose, and its lignin contains more propyl guaiacol units which are prone to condensation reactions involving phenolic hydroxyls. These factors lead to quick depletion of acidic functional groups responsible for chain initiation as described in Table 1. Thus, after just 10-15 minutes of fir oxidation (oxygen consumption 1,5\% based on wood mass), degenerate branching assumes the dominant role in further chain initiation.

Comparison of kinetic parameters for oxidation of fir and aspen wood in alkaline aqueous media by oxygen at low conversion (oxygen consumption below $60 \mathrm{~g}$ per $\mathrm{kg}$ of wood). $\mathrm{pH} 8-12,5$, temperature $70-120^{\circ} \mathrm{C}$

\begin{tabular}{|c|c|c|c|}
\hline Kinetic parameters & \multicolumn{2}{|c|}{ Fir wood } & Aspen wood $[6,17]$ \\
\hline Reaction order in hydroxyl ion & \multicolumn{2}{|c|}{$0,13 \pm 0,02$} & $0,48 \pm 0,02$ \\
\hline Reaction order in oxygen & $\begin{array}{c}\text { Initial stage } \\
0,49 \pm 0,05\end{array}$ & $\begin{array}{c}\text { Final stage }^{* *} \\
0,17 \pm 0,03\end{array}$ & $0,56 \pm 0,04$ \\
\hline Chain initiation & \multicolumn{3}{|c|}{$\mathrm{ROH}+\mathrm{OH}^{-} \rightarrow \mathrm{RO}^{-}+\mathrm{H}_{2} \mathrm{O}$} \\
\hline Degenerate branching & $\begin{array}{r}\mathrm{RO} \\
\mathrm{ROOH}\end{array}$ & $\begin{array}{l}\cdot+\ldots \\
+\ldots(1-4)\end{array}$ & insignificant \\
\hline Kinetic equation $^{* * *}$ & $\begin{array}{r}\text { Non- } \\
\mathrm{W}\left(\mathrm{O}_{2}\right)_{1}= \\
\mathrm{Br} \\
\mathrm{W}(\mathrm{C} \\
\\
\mathrm{W}\left(\mathrm{O}_{2}\right) \\
0 \\
0\end{array}$ & $\begin{array}{l}\text { process } \\
{\left[\mathrm{O}_{2}\right]^{1 / 2}[\mathrm{~L}]^{3 / 2}} \\
\text { ocess } \\
]^{0}\left[\mathrm{O}_{2}\right]^{0} \\
\left.\mathrm{~W}\left(\mathrm{O}_{2}\right)_{2}\right) \\
1 / 2 ; \\
1 / 2\end{array}$ & $\begin{array}{c}\text { Non-branching process } \\
\mathrm{W}\left(\mathrm{O}_{2}\right)_{1}=\mathrm{k}_{1}\left[\mathrm{OH}^{-}\right]^{1 / 2}\left[\mathrm{O}_{2}\right]^{1 / 2}[\mathrm{~L}]^{3 / 2}\end{array}$ \\
\hline
\end{tabular}

" oxygen consumption below 15-25 g per kg of wood; ${ }^{* *}$ oxygen consumption above $15--25 \mathrm{~g} / \mathrm{kg} ;{ }^{* * *}$ [L] is substrate (lignin) concentration 
Fir extractives comprise mainly terpenoid compounds that are mostly chemically inert in the reaction media and have no noteworthy properties in terms of participation in radical chain oxidation. However, their presence in reaction mixtures has dramatic effect on the oxidation process: Kinetics of extracted fir oxidation correspond to non-branching mechanism, while non-extracted fir is oxidized according to degenerate branching laws. This considerable influence of fir extractives can be explained by their ability to form hydrophobic regions in reaction media that are inaccessible to aqueous alkali. It is known that high alkalinity leads to predominantly non-radical destruction of organic hydroperoxides, that accumulate during oxidation. Hydroperoxides destroyed like this do not contribute to oxidation chain branching. However, hydroperoxides protected by hydrophobic extractives are free to decompose homolytically, thus leading to degenerate branching.

The apparent activation energy of fir wood oxidation is practically unaffected by the addition of copper oxide catalyst. This suggests that in both cases there is similar process mechanism, degenerate branching driven by hydroperoxide homolytic cleavage. Comparison of literature data on the rates of catalytic and non-catalytic homolytic cleavage of hydroperoxides indicates that even when extraneous catalyst is not deliberately added to the system, the principal pathway of hydroperoxide cleavage is still catalytic (in this case, it is catalyzed by impurities of transition metals present in the ash portion of wood and/or etched from the reactor walls).

As the concluding remark, a hypothesis concerning the influence of softwood extractives on vanillin formation can be formulated. Vanillin formation is not a chain reaction and requires high alkalinity [18]. I.e., formation of this product is independent of radical chain initiation driven by homolytic hydroperoxide cleavage that primarily occurs in the alkali-excluding regions protected by softwood extractives. The same exclusion of alkali from these regions creates unfavorable conditions for vanillin formation therein. The vanillin that has already formed will accumulate in the highly basic aqueous phase in dissociated form. Therefore, it will be unaffected (not involved in oxidation) by the chain reactions that predominantly take place in the terpenoid phase. So, combining the data on fir extractives' role in softwood oxidation as obtained in the present work together with literature data on vanillin formation in such oxidative processes suggests that the presence of fir extractives in the reaction medium should not considerably affect the yield of vanillin from wood oxidation. This means that the recently proposed oxidative one-stage processing of extracted pine wood into vanillin and cellulose suitable for enzymatic hydrolysis—with high yield in both products—-should be equally suitable for processing non-extracted wood.

\section{References}

1. Hermer E.I. Izvestia vuzov. Lesnoy zhurnal, 2006, no. 6, pp. 94-102. (in Russ.).

2. Tarabanko V.E., Kaygorodov K.L., Skiba E.A., Tarabanko N., Chelbina Yu.V., Baybakova O.V., Kuznetsov B.N., Djakovitch L. Journal of Wood Chemistry and Technology. 2016.. Published online 02 Nov. 2016. Pp. 1-9. DOI 10.1080/02773813.2016.1235583.

3. Wong Z.J., Chen K.F., Li J. Bioresources, 2010, vol. 5, no. 3, p. 1509-1516.

4. Deyneko I.P., Slonim V.Z., Nikolsky S.N., Zarubin N.Ya. Khimia drevesiny, 1983, no. 5, pp. 25-31. (in Russ.).

5. Knill C.J., Kennedy J.F. Carbohydrate Polymers, 2003, vol. 51, no. 3, pp. 281-300.

6. Tarabanko V.E., Pervishina E.P., Hendogina Yu.V. Reaction Kinetics and Catalysis Letters, 2001, vol. 72, no. 1, pp. 153-162.

7. Tarabanko V.E., Koropatchinskaya N.V., Kudryashev A.V., Kuznetsov B.N. Russian Chemical Bulletin, 1995, vol. 44, no. 2, pp. 367-371.

8. Fengel D., Wegener G. Wood. Chemistry, Infrastructure, Reactions. Walter de Gruyter Berlin; New York, 1984, 613 p.

9. Solvent extractives of wood and pulp. TAPPI standard test method T $204 \mathrm{~cm}-97.1997 .4 \mathrm{p}$.

10. Tarabanko V.E., Fomova N.A., Kuznetsov B.N., Kudryashev A.V., Ivanchenko N.M. Reaction Kinetics and Catalysis Letters, 1995, vol. 55, no. 1, pp. 161-170.

11. Sharkov V.I., Kuybina N.I. Khimia gemitselluloz. [Chemistry of hemicelluloses]. Moscow, 1972. 440 p. (in Russ.).

12. Hermer E.I., Butko Yu.G. Khimia drevesiny,. 1983, no. 5, pp. 32-37. (in Russ.).

13. Denisov E.T. Konstanty skorosti gomoliticheskikh zhidkofaznykh reaktsii. [The rate constants of homolytic liquid phase reactions]. Moscow, 1971, p. 51. (in Russ.).

14. Skibida I.P., Sakharov A.M., Emanuel O.N. Itogi nauki i tekhniki, seria "Kinetika i kataliz", 1986, vol. 15, pp. 110-244. (in Russ.).

15. Hermer E.I. Khimia drevesiny, 1992, no. 4, pp. 46-55. (in Russ.).

16. Denisov E.T. Kinetika gomogennykh khimicheskikh reaktsii [Kinetics of homogeneous chemical reactions]. Moscow, 1988. 391 p. (in Russ.).

17. Tarabanko V.E., Pervishina E.P., Nevkritova T.A., Pen R.Z. Khimia rastitelnogo syr'ya, 1999, no. 4, pp. 53-59. (in Russ.).

18. Tarabanko V.E., Petukhov D.V., Selyutin G.E. Kinetics and Catalysis, 2004, vol. 45, no. 4, pp. 569-577. 
